\title{
Síndrome hemorrágica pulmonar em cão associada à leptospirose
}

\author{
Pulmonary Haemorrhagic Syndrome in Dog Associated with Leptospirosis \\ Vinícius Bentivóglio Costa Silva', Simone Tostes de Oliveira Stedile², \\ Renato Silva de Sousa ${ }^{2}$ \& Marlos Gonçalves Sousa ${ }^{2}$
}

Background: Leptospirosis is probably the most widespread and prevalent zoonosis in the world, being classified as an emerging infectious disease for humans and dogs. Sporadically, dogs may present with cough and dyspnea, indicative of pulmonary involvement, associated with interstitial pneumonia and pulmonary hemorrhage with alveolar consolidation. Such changes stem from pulmonary haemorrhagic syndrome, which has emerged as a fatal complication, being documented in some areas in Europe and little described in North America. In this sense, the present study aims to report pulmonary hemorrhagic syndrome in a dog with leptospirosis.

Case: A dog with no defined breed, aged 45 days, weighing $2.2 \mathrm{~kg}$, with a history of apathy, anorexia, jaundice, hematochezia and vocalisation with a two day evolution was seen. Physical examination revealed a rectal temperature of $37.2^{\circ} \mathrm{C}$, icteric mucosa, capillary filling time of two seconds, respiratory rate of 80 movements per minute and heart rate of 140 beats per minute, dehydration rate estimated at $8 \%$, prostration, adequate body score, normal cardiac sounds and clean lung fields, in addition to petechiae in the abdominal region, whose palpation evidenced the presence of fluid in intestinal loops. The blood sample sent to the macroscopic serum agglutination was reagent for the serovar Icterohaemorragiae, titration of 200. Blood count revealed leukocytosis due to neutrophilia, with left-sided deviation, eosinopenia, presence of rare hypersegmentated neutrophils, rare toxic neutrophils, mild cytoplasmic basophilia and rare reactive lymphocytes. Platelet estimation demonstrated thrombocytopenia. No haemoparasites were seen. Regarding the biochemical evaluation, there were changes in liver enzymes and markers of renal failure. Fluid therapy was used with $0.9 \% \mathrm{NaCl}$ solution, $5 \mathrm{mg} /$ $\mathrm{kg}$ doxycycline intravenously every $12 \mathrm{~h}$ and nasogastric probe for administration of nutritional support. One day after the initial evaluation, hemoptysis and diffuse crackling occurred in pulmonary lobes on auscultation. In the radiograph of the chest, pulmonary fields were characterized by a diffuse interstitial bronchial pattern and a focal area, located in the left caudal lobe, with opacification tending to the alveolar pattern. Due to pulmonary alterations, the animal presented respiratory arrest and evolved to death. During necropsy, the macroscopic evaluation revealed a pinkish coloration pattern, with multifocal reddish areas with coalescent interspersed in the parenchyma and hypocrepitation. Regarding the morphology, multifocal hemorrhagic pneumonia was observed, focally extensive, moderate to severe.

Discussion: Recently, pulmonary haemorrhagic syndrome has emerged as a severe form of leptospirosis in many species, including humans and dogs. Patients may develop fulminant pulmonary haemorrhage and result in a high mortality rate. Physiopathogenesis is poorly understood, however, it is believed that there is a multifactorial pathogenesis involving factors related to both pathogen and host, such as immunological mechanisms and coagulopathies. Studies in humans have shown a better evolution after the use of cyclophosphamide, but the benefits of this therapy have not yet been determined in dogs. Therefore, pulmonary haemorrhagic syndrome should be considered in patients with leptospirosis who show respiratory changes, due to the severity of the clinical signs and the high lethality associated with this clinical condition.

Keywords: clinical medicine, infectious diseases, pathological anatomy, pulmonary hemorrhage. Descritores: clínica médica, enfermidades infecciosas, anatomopatologia, hemorragia pulmonar. 


\section{INTRODUÇÃO}

A leptospirose é, provavelmente, a zoonose mais difundida e prevalente no mundo [21], sendo classificada como uma doença infecciosa emergente para humanos e cães [2]. Trata-se de uma enfermidade provocada por espiroquetas do gênero Leptospira, as quais são divididas em bactérias patogênicas e saprófitas [1].

Diversos elementos influenciam a prevalência da infecção por leptospiras em cães, tais como os índices pluviométricos e a presença de roedores na região [6]. Uma vez infectados, os cães desenvolvem doença de intensidade variável, dependendo do sorovar infectante, localização geográfica e resposta imune do hospedeiro, manifestando-se nas formas aguda, crônica ou subclínica [9].

Alguns animais apresentam sinais leves, enquanto outros desenvolvem enfermidade grave, chegando ao óbito [18]. As manifestações clínicas mais comuns da leptospirose em cães são inespecíficas e incluem êmese, letargia e anorexia. Todavia, esporadicamente, esses podem apresentar tosse e dispneia, indicativas de comprometimento pulmonar, associadas à pneumonia intersticial e hemorragia pulmonar com consolidação alveolar, causadas por sorovares mais patogênicos como Icterohaemorragiae, tornando o prognóstico mais reservado [10]. Conforme o último Consenso Europeu sobre leptospirose, tais alterações decorrem da síndrome hemorrágica pulmonar, que tem emergido como uma complicação fatal, sendo documentada em algumas áreas na Europa e pouco descrita na América do Norte [16].

Nesse sentido, o presente estudo tem por propósito relatar a síndrome hemorrágica pulmonar em um cão com leptospirose.

\section{CASO}

Foi atendido no Hospital Veterinário da UFPR um cão, sem raça definida, com idade de 45 dias, pesando 2,2 kg, com histórico de apatia, anorexia, icterícia, hematoquezia e vocalização com evolução de dois dias. Segundo o proprietário, o animal vivia em um campo de futebol e convivia com outros cães da mesma ninhada aparentemente saudáveis, exceto a mãe do filhote, a qual também foi internada, frente histórico de sialocele. Abrigavam-se em local construído com madeira, onde a ração era distribuída, sendo que parte caía no chão e não era recolhida. Quando questionado sobre a possível presença de ratos na região, afirmou ter encontrado um exemplar morto há três dias antes da consulta. A vacinação e a vermifugação não tinham sido realizadas até então.

Ao exame físico, o cão apresentou temperatura retal de $37,2^{\circ} \mathrm{C}$, mucosas ictéricas, tempo de preenchimento capilar de dois segundos, frequência respiratória de 80 movimentos por min e cardíaca de 140 batimentos por min, grau de desidratação estimado em $8 \%$, prostração, escore corporal adequado, bulhas cardíacas normorrítmicas e normofonéticas e campos pulmonares limpos. Ademais, foram identificadas petéquias em região abdominal, cuja palpação evidenciou presença de líquido em alças intestinais.

Ato contínuo, obteve-se amostra sanguínea destinada à avaliação laboratorial. Foram solicitados hemograma, testes bioquímicos (creatinina, ureia, alanina aminotransferase, fosfatase alcalina, proteína total e frações, lactato, bilirrubina total e frações) e sorologia para leptospirose por soroaglutinação microscópica (SAM). Na SAM foram testados os sorovares Autumnalis, Bratislava, Canicola, Copenhageni, Cynopteri, Grippotyphosa, Hardjo, Icterohaemorrhagiae, Pomona e Pyrogenes, sendo considerados reagentes títulos $\geq 100$. Dentre os resultados obtidos, o hemograma revelou leucocitose por neutrofilia, com desvio à esquerda, eosinopenia, presença de raros neutrófilos hipersegmentados, raros neutrófilos tóxicos, leve basofilia citoplasmática e raros linfócitos reativos. A estimativa de plaquetas demonstrou trombocitopenia. Não foram visualizados hemoparasitos. No que concerne à avaliação bioquímica, observou-se elevação das enzimas alanina aminotransferase e fosfatase alcalina, além de hiperbilirrubinemia e azotemia (creatinina e ureia aumentadas). A amostra enviada para a SAM apresentou-se reagente apenas para o sorovar Icterohaemorragiae, com titulação de 200.

Diante desses resultados, instituiu-se, como terapia nosocomial, fluidoterapia com solução de $\mathrm{NaCl}$ 0,9\% (374 mL/dia, baseada na reposição, manutenção e perdas contínuas) e doxiciclina (Doxiciclina Solução Injetável® $)^{1}$, na dose de $5 \mathrm{mg} / \mathrm{kg}$, por via intravenosa, a cada $12 \mathrm{~h}$. No intuito de manter um aporte nutricional adequado, realizou-se sondagem nasogástrica e administração de Nutralife ${ }^{\circledR}{ }^{1}$, conforme recomendações do fabricante de acordo com o peso, distribuído em um total de 6 refeições ao longo do dia. Visto à hipotermia, o paciente foi aquecido com bolsas de água morna até completa normalização da temperatura. 
Um dia após a avaliação inicial, o paciente apresentou nítida piora do quadro clínico, exibindo, inclusive, hemoptise e crepitação difusa em lobos pulmonares na ausculta. Para tanto, solicitou-se avaliação radiográfica do toráx, cujas imagens evidenciaram campos pulmonares caracterizados por padrão in-
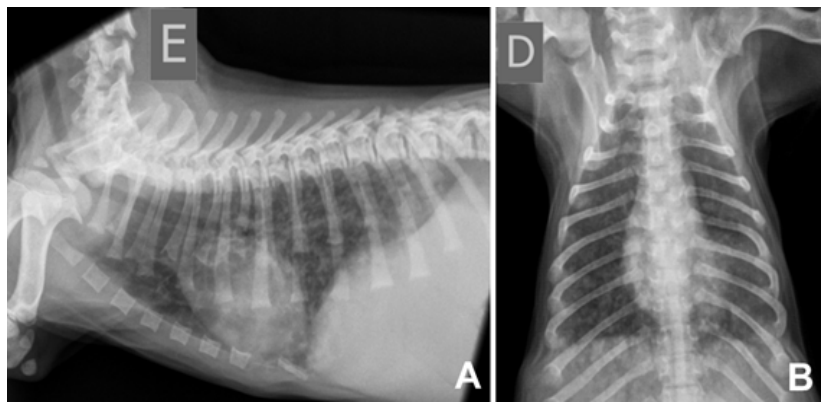

Figura 1. Cão, SRD, de 45 dias, imagens radiográficas torácicas. A- Projeção latero-lateral esquerda evidenciando área focal de opacificação tendendo a alveolar em lobo caudal esquerdo. BProjeção ventro-dorsal mostrando campos pulmonares com severa opacificação e padrão intersticiobronquial de forma difusa.

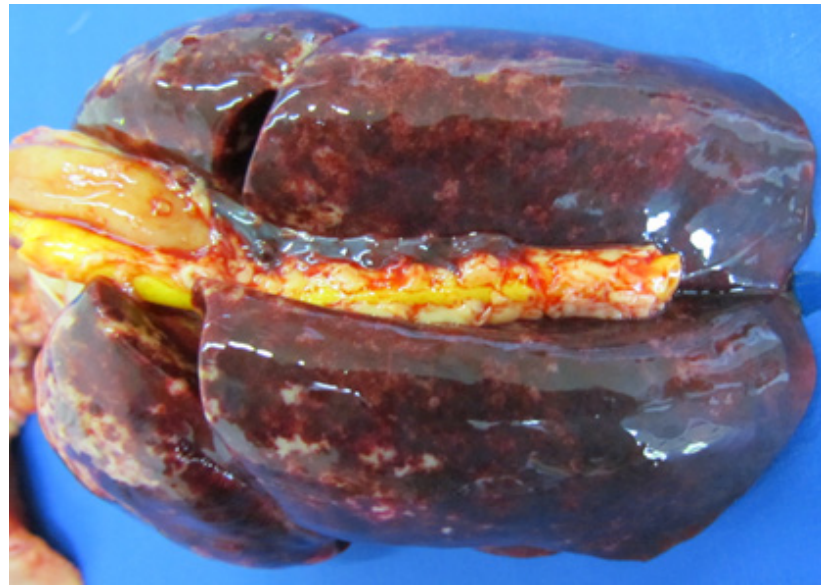

Figura 2. Cão, SRD, de 45 dias. Hemorragia pulmonar multifocal a coalescente, acentuada.

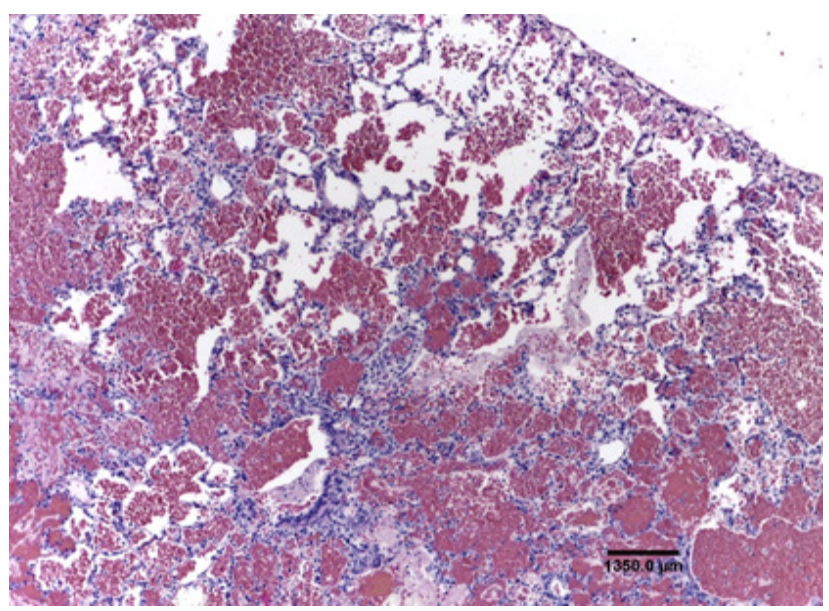

Figura 3. Cão, SRD, de 45 dias. Hemorragia pulmonar intraalveolar difusa e acentuada. HE, obj.10x. [Bar $=1350 \mu \mathrm{m}]$. tersticiobronquial de forma difusa e com área focal, localizada em lobo caudal esquerdo, de opacificação tendendo ao padrão alveolar (Figura 1). Devido às alterações pulmonares, apresentou parada respiratória e evoluiu ao óbito.

Durante a necropsia, a avaliação macroscópica evidenciou coloração amarela difusa e bastante acentuada no tecido subcutâneo. Os pulmões, por sua vez, apresentavam padrão de coloração rósea, com áreas avermelhadas multifocais a coalescentes entremeadas no parênquima e hipocrepitação (Figura 2). O fígado exibia coloração castanha difusa, acentuada. Nos rins, notou-se coloração amarela intensa, difusa.

Foram coletadas amostras dos pulmões, fígado, baço, encéfalo, rins, estômago, intestinos, pâncreas e vesícula urinária, sendo fixados em formol tamponado $10 \%$, depositados em frascos individuais e rotulados, onde permaneceram por um período mínimo de $24 \mathrm{~h}$. Os fragmentos de tecido foram processados rotineiramente para avaliação histopatológica, cortados em micrótomo rotativo ( $4 \mu \mathrm{m}$ de espessura) e corados com hematoxilina e eosina (HE).

No que tange à morfologia, observou-se pneumonia hemorrágica multifocal a focalmente extensa, moderada a acentuada (Figura 3). No tecido hepático, dissociação difusa e leve de cordões de hepatócitos acompanhada por moderada retenção biliar, vacuolização hepatocelular leve a moderada e discretos focos de necrose de coagulação de hepatócitos. Baço e encéfalo exibiam congestão difusa, leve a moderada. Os rins exibiam nefrite linfoplasmocitária multifocal e leve, degeneração tubular, multifocal, leve a moderada, mineralização multifocal e discreta, além de congestão difusa e leve. No estômago e intestino havia mineralização multifocal leve e, no pâncreas, congestão difusa e leve. Ademais, notou-se hemorragia multifocal e moderada da camada muscular em vesícula urinária.

\section{DISCUSSÃO}

Recentemente, a síndrome hemorrágica pulmonar emergiu como uma forma severa de leptospirose em muitas espécies, incluindo seres humanos e cães. Os pacientes podem desenvolver hemorragia pulmonar fulminante e consequente alta taxa de mortalidade. A fisiopatogenia é mal compreendida, todavia, acredita-se que haja uma patogênese multifatorial envolvendo fatores relacionados tanto ao patógeno quanto ao hospedeiro, como mecanismos imunológicos e coagulopatias [14,20]. 
A exposição dos cães à Leptospira spp. presente no ambiente ocorre por meio do contato com a urina de um hospedeiro infectado, água contaminada ou solo úmido, onde as bactérias têm a capacidade de sobreviver durante vários meses [15]. As leptospiras penetram o organismo através das mucosas conjuntival, nasal, oral ou genital, bem como a pele com escoriações. Uma vez na corrente sanguínea, distribuem-se em órgãos parenquimatosos, como fígado, baço, rins e, ocasionalmente, meninges [6]. O paciente ora descrito vivia em um local inapropriado, com diversos fatores predisponentes à infecção por lepstospiras, tais como ração disposta sem recolhimento das sobras e consequente presença de roedores.

A infecção pelo sorovar Icterohaemorrhagiae geralmente desencadeia óbito nas primeiras $48 \mathrm{~h}$, no entanto os cães sobreviventes a esse período podem desenvolver a síndrome icterohemorrágica, caracterizada por prostração, icterícia e hemorragias difusas, as quais são marcantes especialmente em parênquima pulmonar e trato gastrintestinal, embora lesões hepáticas também sejam observadas [13], em consonância ao observado no animal aqui descrito.

Na fase aguda da enfermidade, as manifestações clínicas são típicas da síndrome de resposta inflamatória sistêmica associada à sepse, com hipotermia e depressão profunda nos animais terminais, podendo surgir distúrbios hemostáticos [10], tal qual o apresentado pelo cão em questão (trombocitopenia), tendo como resultado, petéquias e hematoquezia.

Sinais respiratórios, como taquipneia e dispneia leve a grave, podem ocorrer em cães com leptospirose, por muitas razões, incluindo edema pulmonar devido à hiperhidratação, pneumonia aspirativa, dor ou acidose. Contudo, os clínicos também devem considerar síndrome hemorrágica pulmonar como causa de dispneia em pacientes com leptospirose, já que essa apresentação clínica pode ser mais comum em cães com leptospirose do que geralmente se acredita [12].

O diagnóstico da leptospirose canina apoia-se nas informações clínico-epidemiológicas, bem como nos exames laboratoriais complementares [7]. Anemia, leucocitose por neutrofilia e trombocitopenia são os achados mais comuns no hemograma. No que concerne ao perfil bioquímico, azotemia, aumento das enzimas hepáticas e hiperbilirrubinemia costumam ser evidenciados [4], tal qual fora identificado no animal em questão. Contudo, a soroaglutinação microscópica, que se baseia na detecção de anticorpos anti-leptospira, é o teste de referência para o diagnóstico sorológico dessa enfermidade. Ainda assim, é importante destacar que os anticorpos anti-leptospira somente poderão ser documentados a partir de sete a dez dias pós-infecção [5,19].

Os exames de imagem são pouco conclusivos para o diagnóstico de leptospirose. No entanto, a radiografia torácica torna-se imprescindível caso haja tosse, cansaço ou dispneia [10]. Nesses casos, as lesões potencialmente encontradas variam de um padrão intersticial leve a retículo-nodular leve a grave, com infiltrados alveolares focais [3]. No paciente avaliado, as alterações radiográficas de tórax corroboram com a literatura que descreve as características imaginológicas da hemorragia pulmonar secundária à leptospirose.

Durante a necropsia, as principais lesões observadas incluíram icterícia e hemorragia, principalmente no parênquima pulmonar. Alterações macroscópicas hepáticas e renais foram frequentes e se caracterizavam principalmente por alteração de coloração. Tais alterações assemelham-se ao descrito em um estudo que avaliou os aspectos anatomopatológicos da leptospirose em 53 cães [15]. Além disso, na avaliação histopatológica dos pulmões, a hemorragia intersticial e alveolar multifocal, de intensidade moderada a acentuada, acompanhada por acentuada deposição de feixes de fibrina, infiltrados neutrofílico e histiocitário multifocal e leve, além de edema leve a moderado e congestão difusa moderada, condizem com os achados descritos na literatura em cães com síndrome hemorrágica pulmonar associada à leptospirose [11].

O tratamento da leptospirose envolve terapia de suporte, utilização de agentes antimicrobianos e cuidados direcionados às manifestações renais e/ou hepáticas. A terapia antibiótica deve ser iniciada tão logo haja suspeita da enfermidade, sendo essencial para eliminar a bacteremia [8]. Penicilinas ou doxiciclina têm sido considerados os antimicrobianos de escolha tanto em seres humanos quanto em cães com leptospirose [17].

Nos pacientes com síndrome hemorrágica pulmonar associada à leptospirose, pode ser necessário oxigenioterapia ou até mesmo ventilação mecânica em casos mais severos. Estudos em seres humanos demonstraram melhor evolução após uso de ciclofosfamida, porém os benefícios dessa terapia ainda não foram determinados em cães. Por outro lado, o tratamento de seres humanos e cães que apresentam complicação respiratória com dexametasona e desmopressina não apresentou benefícios [18]. 
V.B.C. Silva, S.T.O. Stedile, R.S. Sousa \& M.G. Sousa. 2017. Síndrome hemorrágica pulmonar em cão associada à leptospirose.

Acta Scientiae Veterinariae. 45(Suppl 1): 240.

Destarte, a síndrome hemorrágica pulmonar deve ser considerada em pacientes com leptospirose que apresentem alterações respiratórias, haja vista a severidade dos sinais clínicos e a alta letalidade associadas à essa condição clínica.
MANUFACTURER

${ }^{1}$ Vetnil Indústria e Comércio de Produtos Veterinários. Louveira, SP, Brazil.

Declaration of interest. The authors report no conflicts of interest. The authors alone are responsible for the content and writing of the paper.

\section{REFERENCES}

1 Adler B. \& Moctezuma A.P. 2010. Leptospira and leptospirosis. Veterinary Microbiology. 140(3-4): 287-296.

2 Alton G.D., Berke O., Reid-smith R., Ojkic D. \& Prescott J.F. 2009. Increase in seroprevalence of canine leptospirosis and its risk factors, Ontario 1998-2006. Canadian Journal of Veterinary Research. 73(3): 167-175.

3 Baumann D. \& Fluckiger M. 2001. Radiographic findings in the thorax of dogs with leptospiral infection. Veterinary Radiology \& Ultrasound. 42(4): 305-307.

4 Brown C.A., Roberts A.W., Miller M.A., Davis D.A., Brown S.A., Bolin C.A., Jarecki-Black J., Greene C.E. \& MillerLiebl D. 1996. Leptospira interrogans serovar grippotyphosa infection in dogs. Journal of American Veterinary Medical Association. 209(7): 1265-1267.

5 Chappel R.J., Goris M., Palmer M.F. \& Hartskeerl R.A. 2004. Impact of proficiency testing on results of the microscopic agglutination test for diagnosis of leptospirosis. Journal of Clinical Microbiology. 42(12): 5484-5488.

6 Faine S., Adler B., Bolin C. \& Perolat P. 1999. Leptospira and leptospirosis. 2nd edn. Melbourne: MediSci, 272p.

7 Goldstein R.E., Lin R.C., Langston C.E., Scrivani P.V., Erb H.N. \& Barr S.C. 2006. Influence of infecting serogroup on clinical features of leptospirosis in dogs. Journal of Veterinary Internal Medicine. 20(3): 489-494.

8 Goldstein R.E. 2010. Canine leptospirosis. The Veterinary Clinics of North America: Small Animal Practice. 40(6): 1091-1101.

9 Hagiwara M.K., Lustosa M. \& Kogika M.M. 2004. Leptospirose canina. Vet News. 11(67): 7-8.

10 Jericó M.M., Andrade Neto J.P. \& Kogika M.M. 2015. Tratado de medicina interna de cães e gatos. Rio de Janeiro: Roca, $1238 \mathrm{p}$.

11 Klopfleisch R., Kohn B., Plog S., Weingart C., Nöckler K., Mayer-Scholl A. \& Gruber A.D. 2010. An emerging pulmonary haemorrhagic syndrome in dogs: similar to the human leptospiral pulmonary haemorrhagic syndrome? Veterinary Medicine International. 2010: 1-7.

12 Kohn B., Steinicke K., Arndt G., Gruber A.D., Guerra B., Jansen A., Kaser-Hotz B., Klopfleisch R., Lotz F., Luge E. \& Nöckler K. 2010. Pulmonary abnormalities in dogs with leptospirosis. Journal of Veterinary Internal Medicine. 24(6): 1277-1282.

13 Levett P.N. 2001. Leptospirosis. Clinical Microbiology Reviews. 14(2): 296-326.

14 Medeiros F.R., Spichler A. \& Athanazio D.A. 2010. Leptospirosis-associated disturbances of blood vessels, lungs and hemostasis. Acta Tropica. 115(1-2): 155-162.

15 Raghavan R., Brenner K., Higgins J., Van Der Merwe D. \& Harkin K.R. 2011. Evaluations of land cover risk factors for canine leptospirosis: 94 cases (2002-2009). Preventive Veterinary Medicine. 101(3-4): 241-249.

16 Schuller S., Francey T., Hartmann K., Hugonnard M., Kohn B., Nally J.E. \& Sykes J. 2015. European consensus statement on leptospirosis in dogs and cats. Journal of Small Animal Practice. 56(3): 159-179.

17 Suputtamongkol Y., Pongtavornpinyo W., Lubell Y., Suttinont C., Hoontrakul S., Phimda K., Losuwanaluk K., Suwancharoen D., Silpasakorn S., Chierakul W. \& Day N. 2010. Strategies for diagnosis and treatment of suspected leptospirosis: a cost-benefit analysis. PLoS Neglected Tropical Diseases. 4(2): 1-7.

18 Sykes J.E., Hartmann K., Lunn K.F., Moore G.E., Stoddard R.A. \& Goldstein R.E. 2011. ACVIM small animal consensus statement on leptospirosis: diagnosis, epidemiology, treatment, and prevention. Journal of Veterinary Internal Medicine. 25(1): $1-13$.

19 Teixeira M.A., Gonçalves M.L.L., Riediger I.N., Prosser C.S., Silva S.F.C., Biesdorf S.M., Mosko P.R.E., Morais H.A. \& Biondo A.W. 2008. Sorologia negativa e PCR positiva: a importância da biologia molecular para o diagnóstico de leptospirose aguda em um cão. Clínica Veterinária. 8(73): 44-48.

20 Tochetto C., Flores M.M., Kommers G.D., Barros C.S.L. \& Fighera R.A. 2012. Aspectos anatomopatológicos da leptospirose em cães: 53 casos (1965-2011). Pesquisa Veterinária Brasileira. 32(5): 430-443.

21 World Health Organization. 1999. Leptospirosis worldwide. Weekly Epidemiological Record. 74(29): 237-242.

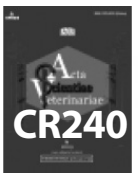

